\title{
I $\left.\mathbf{n} \mathbf{h} \mathbf{a} \mathbf{l} \mathbf{t}^{\prime}\right)$.
}

\author{
Brster Absclınitt.
}

A l l g e m e i n e Ph y s i k.

\section{Molecularphysik.}

Drafosse. Ueber die Natur der Hemiedrie und ilıre Beziehungen zu den phyikalischen Eigenschaften der Krystalle . • 3

A. Gaudin. Ueber die Gruppirung der Atome in den Moleculen und die wahren Ursachen der Krystallformen . . 4

- Bildung der Krystalle aus Molecularpolyedern . . 4

J. A. D. Anordnung der Atome in Flüssigkeiten . . $\quad 5$

CARrol. Anordnung der Atome in Flüssigkeiten . . 5

Purgold. Von den Krystallen und ihrer Entstehung . $\quad 5$

P. Kremers. Ein Vergleich zwischen der Modification der unittleren specifischen Wärme und des mittleren Volums . $\quad 6$

- Ueber die Schmelz- und Siedepunkte der Glieder ein-

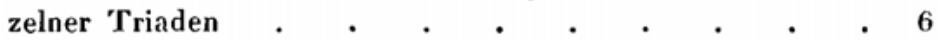

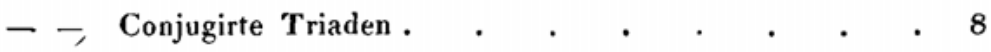

H. Kopp. Ueber die Volume flüssiger Verbindungen . 8

C. S. Schönвern. Zusammenhang der katalytischen Erscheinun-

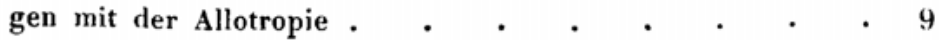

Osans. Versuche über den Ozonwasserstoff. . . . . 14

Berthвцот. Untersuchungen über den Schwefel . . . 15

- Ueber die Bildung des unlöslichen Schwefels bei Einwirkung der Wärme . $\quad . \quad$ • $\quad$. 20

1) Ueber mit einem Sternchen $\left({ }^{*}\right)$ bezeichneten Aufsätze ist keiu Bericht erstattet. 
Вевтнецот. Ueber den weichen Schwefel der Hyposulfite . 22

C.S. C. Deville. Ueber die Eigenschaften des Schwefels . 22

J. W. Malcet. Ueber den rothen Scliwefel . . . . 23

R. Weber. Ueber die Wärmeentwickelung beí Molecularveränderungen des Schwefels und des Quecksilherjodids . . 23

R. Napolr. Prioritätsanspruch auf die Entdeckung des rothen Phosphors . . . . . . . . . . . .

F. Wöhlkr und H. S. C. Drfrlce. Ueber das Bor, Analyse desselben und physikalische Eigenschaften. . . . 25

II. S. C. Deville. Ueber das Silicium . . . . . . 26

J. Hausmans. Ueber die durch Molecularbewegungen in starren leblosen Körpern bewirkten liormveränderungen. Zweite Abhandlung . . . . . . . . . . . 28

J. Henry. Unrifs eines Vortrages über Pliysik . . . . 29

F. Redtenbacheh. Dynamidensystem . . . . . . 31

A. E. Nordenskı̈̈цd. Dichtigkeit chemischer Verbindungen theoretisch berechnet . . . . . . . . . . . 40

2. Adhăsiou.

J.Stennouse. Ueber entfärbende Kolıle und ihr Vermögen einige Gase zu absorbiren $\quad . \quad$. $\quad . \quad$. $\quad$. 42

G. Buisr. Ueber die Ursache der Erscheinung, dafs die Federn des Wasserhulıns und die Blätter von Pflanzen vom Wasser niclit benetzt werden. . . . . . . 46

C. MÊxe. Anwendung des Thonerdehydrats zur Entfärbung, an

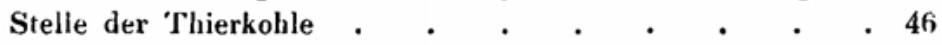

Grandr. Verfahren Zeichnungen, Kupferstiche etc. zu repro-

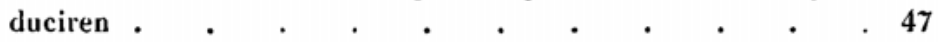

C. Cessner und Kretzinsky. Ueber die Anwendung des Thonerdehydrats als Entfärbungsmittel für alle Gattungen von $\mathrm{Me}$ lassen, Colonial- und Rübenrohrzucker . . . .

3. Capillarität.

C. Wolfr. Einflufs der Temperatur auf die Erscheinungen in

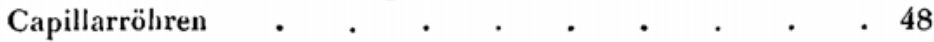

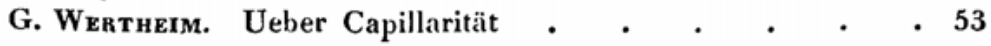

C. A. VA Lson. Ueber die Erscheinungen der Capillarität . . 57

E. Drsarss. Uelser die capillare Steighöhe des Wassers zwischen zwei parallelen Wänden . . . . . . . . 61

Gıвект. Zur Theorie der Capillarphänomene. $\quad . \quad 62$

J. C. Fonтone. Zur mathematischen Theorie der Capillaritait . 63 
${ }^{*}$ A. Dawidop. Ueber die Theorie der Capillaritätserschei-

Seite nungen .

4. Diffusiou.

A. Lieben. Homogenität der Lösungen . . • . . 63

А. Fıк. Erwiederung auf einige Stellen der Abhandlung; „Ueber

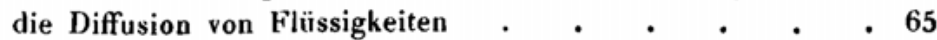

W. Schmidt. Versuche über die Endosmose des Glaubersalzes 65

T. Simmler und H. Wrid. Ueber einige Methoden zur Bestimmung der bei der Diffusion einer Salzlösung in das reine Lösungsmittel auftretenden Constanten . . . . . 68

Maggiorani. Ueber die Endosmose des Eiweifses . 69

\section{Dichtigkeit.}

Jocly. Ueber die Physik der Molecularkräfte . . . . 70

H. Kopp. Berechnung der Dichtigkeiten des Dampfes • • 72

H. S. C. Devirle und L. Troost. Ueber die Dampfdichte gewisser mineralischer Substanzen. . . . . . . . . 73

W. Knop. Einige Bemerkungen über die bei Angaben von Dichten oder specifischen Gewichten von Gasen und Dämpfen

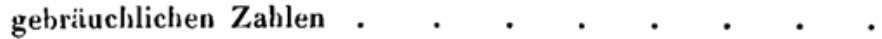

Воврксквк. Relationen zwischen Dichtigkeit, specifischer Wärme und Zusammensetzung der Gase . . . . . . 75

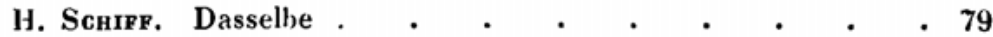

P. Кнемеrs. Ueber die Aenderungen, welche die Modification des mittleren Volums gelöster Salzatome durch die Aenderung der 'Temperatur erleidet

J. Nasmrтh. Erscheinungen die bei geschmolzenen Substanzen

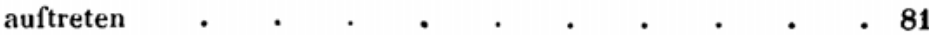

'Th. Andrews und P. G. FAIt. Dichtigkeit des Ozons . . 82

Lenz. Ueber den Gebrauch des FAhrenherx'schen Aräometers zur Bestimmung des Salzgehaltes der Meerwasser. . . 82

Vogel und Rerschauer. Ueber die specifischen Gewichtsbestimmungen von Flüssigkeiten . . . . . . .

A. Erman. Untersuchungen über den Salzgehalt des Meerwassers und dessen Werth im mittelländischen und atlantischen

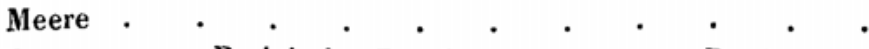

R. Концrausch. Praktische Regeln zur genaueren Bestimmung des specifischen Gewichts

6. Maass und Messen.

B. Arny. Ueber den neuen englischen Normalmaafsstab. 
L. Ruau, Note über ein Aräometer. . . . . . . . 91

v. KoвEll. Neue Methode, Krystallwinkel zu inessen . . 91

F. Prafr. Ueber die Messung der ebenen Krystallwinkel und deren Verwerthung für die Ableitung der Flächen . . 91

R. Wolf. Die Erfindung der Röhrenlibelle . . . . 92

Laugrar. Versuche über die Empfindlichkeit des Auges bei

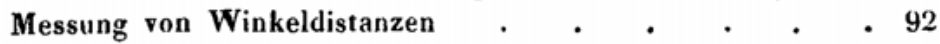

* Deramorinik̀re und Sḱgurer. Ueber eine neue Form des Gewichts, die dieselbe bei allen Gewichten bleibt . . . 93

*Bkranger und Comp. Wägeapparat . . . . . . 93

* A. D'Aвbadre. Decimalsystem der Maaf'se . . . . . 93

* Perreaux. Vergleichinstrument zur Berichtigung der Meterinaafsstäbe . . . . . . . . . . . 93

\section{Mechanik.}

О. Sснцӥмгсн. Ueber die analytischen Beweise des Satzes voın Parallelograınıne der Kräfte . . . . . . . . . . 93

E. Lamarce. Ueber den Begriff und die innere Natur der Ge-

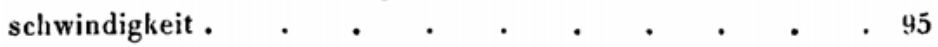

C. KüPPER. Zur Theorie der Trügheitsmomente . . . 95

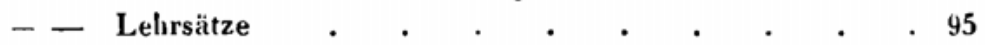

Tн. D'Estoçuors. Ueber die Homologie in der Mechanik . $96^{\circ}$

Bhennecke. Lehre vom Wurf . . . . . . . . 96

Ostrogradskr. Ueber die Anwendung der Linearpolynome in

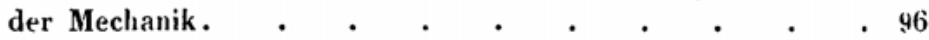

E. Brassine. Ueber die Glieder, die die allgenneine Gleichgewichtsgleichung für den Fall der Reibung vervollständigen 46

Phillyps. Ueber das Princip der kleinsten Action und das D'Alembent'sche Princip in der relativen Bewegung . .

Résal. Ueber die relative Bewegung eines festen Körpers in Beziehung auf ein unveränderliches System . . . . . 47

Faraday. A. Mechanic. B. Cheyerton. Goosequill u. Andere.

- B. Cheverton. A. Mrchanic. Ueber die Erhaltung der

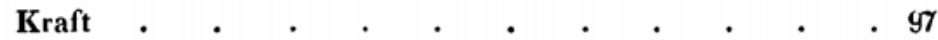

B. Cheverton. E. Brücke. Ueber die Erhaltung der Kraft . 98

Lroxd. Ueber Erhaltung der Kraft . . . • . . . 99

Ostrogradskx. Ueber das Princip der kleinsten Wirkung . 99

Porssor. Abhandlungen über Dynamik. Ueber den Stofs der

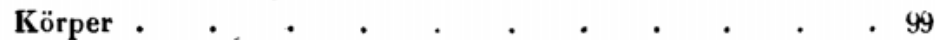

Carlex. Ueber eine Klasse dymanischer Probleme . . . 105 
J. Bertrand. Ueber einzelne einfachste Ausdrücke, die die Integrale der Differentialgleichungen der Bewegung eines materiellen Punktes annehmen können.

Schrlzbach. Ueber die Bewegung eines Punktes auf der Ober-

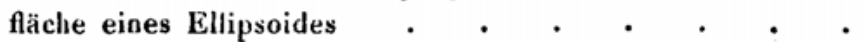

Caxbax. Beweis des Hammton'schen Theorems, betreffend die gleichen Zeiten bei deın Circularhodograph. . . . $10 y$

Brer. Ueber die Enveloppe gewisser Planetenbahnen • 109

A. Caxbay. Bericht über die neueren Fortschritte der theore-

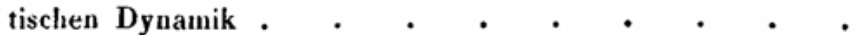

- Ueber die Hamrcton'sche Methode bei Behandlung des Problems von drei und mehr Körpern. . . . .

- Ueber die Lagrange'sche Lösung des Problems von

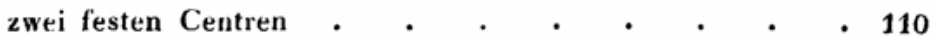

JELlex. Ueber einige, auf die 'Theorie der Attraction bezüg-

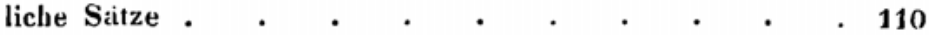

E. J. Rourh. Ueber einen Satz der Attraction . . $\quad 111$

Caxlex. Note über die Curve $\frac{m}{r}+\frac{m_{1}}{r_{1}}=\boldsymbol{C} \cdot$. . $\quad 111$

T. A. Hirst. Körper gleicher Anziehung . . . 111

J. Bounget. Anziehung elliptischer Paraboloide . . 112

J. A. Hrrst. Ueber das Potential einer unendlich dïnnen Schicht, welche von zwei elliptischen Paraboloiden eingeschlossen ist. $. \quad . \quad . \quad . \quad . \quad . \quad . \quad$.

W. SchEIbNer, Fiächenpotential

G. Lejeune-Drhichezt. Ueber eine neue Formel zur Bestinmung der Dichtigkeit einer unendlich dünnen sphärischen

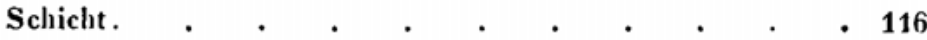

H. G. Lösung eines mechanischen Problems • • . . 117

Hennessy. Ueber die Richtung der Schwęre auf der Erde . 117

Rozer. Ueber die in Schottland beobachtete Ablenkung der Verticalen . . . . . . . . . . . . 118

x. Ueber die Bestimmung der mittleren Erddichtigkeit . 118

W. Jасов. Differenzen in der Dichtigkeitsbestimunung der Erde 118

De Boucheporn. Beinerkung über die Veränderung der Schwere 118

D. Vaughan. Secularveränderung der Mond- und Erdbewegung durch den Einflufs der Fluth . . . . 119

T. Reuleaux. Ueber die Unbestimmtheit des Ausdrucks „Pferdekraft" . . . . . . . . . . . . . . 119

G. Prlanzedrr. Multiplumsbrückenwagen . $\quad . \quad$. 119 
Sснӧngmans. Benutzung der Brückenwagen zur Ermittelung der Geschwindigkeit geschossener und fallender Körper.

Harcess. Besclıreibung der Apparate, welche in seiner Abhandlung ,über die statischen Momente der menschlichen Gliedmaafsen" zur Auffindung der Lage des allgemeinen Schwerpunkts bezeichnet sind . . . . . . .

R. Crausius. Ueber die Entfernungen, in welchen die von einem Eisenbahnzuge bewirkten Erschütterungen noch spürbar sind

G. 'Treviranus. Ueber Ballistik . . . . . . . 121

Zantedeschr. Apparat zur Mittheilung der Bewegung . . 121

J. A. Grunrrt. Ueber die Entwickelung der Grundformeln der Drehung eines Systems materieller Punkte un einen festen Punkt etc. No. VI. Ueber die Hauptaxen eines Systems materieller Punkte

Foucaurt'sche Versuche.

E. Lotrner. Ueber die, der Einwirkung der Schwere entzogenes, aber unter dem Einflufs der Erdbewegung rotirenden Körper, Theorie des Foucaurt'schen Gyroskops . .

J. A. Grunert. Theorie des Foucaurt'schen Pendelversuchs aus neuem Gesichtspunkt dargestellt wit Rücksicht auf die ellipsoidische Gestalt der Erde . . . . . . 122

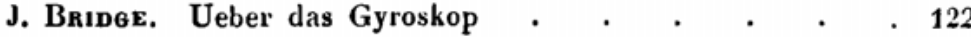

J. G. Bannard. Die sich selbst erhaltende Kraft des Gyroskops analytisch behandelt . $. \quad . \quad . \quad . \quad . \quad . \quad .123$

H. A. Newron. Erklärung der Bewegung des Gyroskops . 123

J. B. Eads. Erklärung der Mechanik des Rotoskops . . 123

W. Соок. Theorie des Gyroskops . . . . . 124 Physiologische Mechanik. Literatur.

\section{Hy dromechanik.}

A. Сиввsch. Ueber eine allgemeine Transformation der hydrodynamischen Gleichungen . . . . . . . . 124

- - Ueber die Bewegung eines Eilipsoids in einer tropfbaren Flüssigkeit . $\quad$. $\quad . \quad$. $\quad$. $\quad$. $\quad$. $\quad$ • 125

L. Matrhisssen. Ueber die Gleichgewichtsfiguren homogener freier rotirender Flüssigkeiten . . . . . . . 125

Berr. Ueber die Prateau'schen Versuche mit Flüssigkeiten, welche der Wirkung der Schwere entzogen sind. . . 126

Dupurt. Ueber die Bewegung des Wassers durch poröse Erdschichten 
Bupf. Schreiben an G. Magnus . . . . . . . 127

Gladstone. Bemerkungen über den Schaum . . . . 127

de Calignx. Beschreibungen der Eigenschaften eines gewöhnlichen Regulators an mehreren hydraulischen Maschinen. . . . . . . . . . . . .

G. Rennre. Experimente zur Bestimmung des Widerstandes einer Schraube, die sich in verschiedenen Tiefen und mit verschiedener Geschwindigkeit im Wasser bewegt $\quad$. 128

H. Darcx. Einige Abänderungen an der Piror'schen Röhre . 128

G. Lejeung-Dirichlet. Untersuchungen über ein Problem der

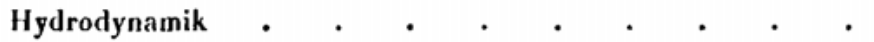

9. Aeromechanik.

Cantor. Physikalische Aufgabe

F. G. Schaffgotsch. Ueber eine Erscheinung bei plötzlich aher schwach geändertem Luftdruck . . . . . .

P. Volpicerrr. Allgemeine Formel für das Manometer mit comprimirter Luft und für das Stereometer. . . . 129

Daru. Mechanische Wirkung der comprimirten Luft . . 129

R. Bunsen und L. Schischrofr. Chemische Theorie des Schiefspulvers. . . . . . . . . . . . .

H. NвımкE. Erfahrungen bei der Sprengarbeit in den Oberharzer Gruben . . . . . . . . .

10. Elasticität fester Körper.

Maнıstre. Ueber die Rotationsgeschwindigkeiten, die gewisse Räder annehmen können, ohne durch die Wirkung der Centrifugalkraft einen Bruch zu erleiden. . . . .

- Ueber die Grenzen der Geschwindigkeiten, die man den Eisenbahnzügen ertheilen kann, ohnen einen Bruch der Schienen befürchten zu brauchen. . . . . . .

R. Hoppr. Biegung prismatischer Stäbe . . . . . 138

J. H. Koosen. Entwickelung der Fundanentalgesetze über die Elasticität und das Gleichgewicht im Innern chemisch homogener Körper $\quad . \quad$. $\quad . \quad$. $\quad$. $\quad$. . . .

Crapeyron. Berechnung der Druckvertheilung eines elastischen Balkens der frei auf ungleich entfernten Stützen liegt .

Bresse. Berechnung des Widerstandes eines Dampfkessels mit schwach elliptischem Profil . . . . . . .

Dahlmans. Absolute Festigkeit verschiedener Eisen- und Stahlhärten . . . . . . . . . . . 
C. F. Dretzer. Die Elasticität des vulcanisirten Kautschuks und Bemerkungen über die Elasticität fester Körper überhaupt. 143

J. Dupuır. Note über den Horizontalschuls der bei Constructionen angewendeten Balken $\quad . \quad$. $\quad . \quad$..$\quad$.

De Saint-Venant. Ueber den transversalen Stofs und den lebendigen Widerstand bei Balken, die an ihren Enden unterstützt sind . . . . . . . . . . . 145

G. Wertheim. Ueber die 'Torsion . . . . . . 145

W. Fairbainn. Festigkeit des Schmiedeeisens bei verschiede-

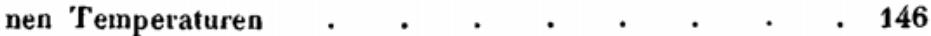

Phiclirs. Ueber „Parachocs” und „Heurtoirs” bei Eisenbahnen 148

Drkvy. Ueber die Anwendung der Theorie von Phrlerps auf die Construction von Locomotivfedern neuer Art . . . 149

E. Hoderinson. Experimentaluntersuchungen über die Festig-

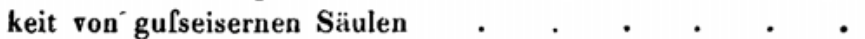

W. H. Barlow. Ueber ein Element der Stärke von Balken, die dem Querdruck unterworfen sind, vom Verfasser genannt „Biegungswiderstand". Zweite Mittheilung . . . .

A. F. Kuprper. Untersuchungen über Elasticität, welche wällrend der Jahre 1850 bis 1855 in dem Petersburger physikalischen Observatorium angestellt wurden . . . .

W. Fairbairs. Ueber die relativen Werthe des Widerstandes verschiedener Steinsorten, den diese gegen die Zusammendrückung ausüben $\quad . \quad$. $\quad$. $\quad$. $\quad$. . . . .

11. Veränderungen des Aggregatzustandes.

A. Gefrieren, Erstarren.

E. Desains. Festwerden von Flüssigkeiten die bis unter ihrem Erstarrungspunkt abgekühlt sind . . . . . .

v. Ваво. Gefrieren des Quecksilbers in einem glühenden 'Tiegel .

B. Schinelzen.

J. Trndals. Einige physikalische Eigenschaften des Eises . 158

F. G. Scнағғgотsch. Ueber zwei ausgezeichnete Beispiele der Schmelzpunktveränderung . . . . . . . . 160

C. Auflösung.

A. Breanu. Bemerkungen über die Auflösungen einiger Carbonate, besonders des kohlensauren Kalks . . . .

*D. Aвacher. Untersuchungen über die wechselseitige Auflösung der Flüssigkeiten. . . . . . . . 1 
D. Absorption.

Seite

L. Mrrer. Die Gase des Blutes . . $\quad$ • $\quad$. 162

E. Prugor. Studien über die Zusammensetzungen der Gewässer 164

v. Baвo. Absorption des Wasserdampfes durch die Ackererde. 165

E. Sieden, Verdampfen.

v. Ваво. Ueber die Spannkraft des sich aus Salzlösungen entwickelnden Wasserdampfes . . . . . . . . 165

F. Leidenfrost's cher Versuch.

Zweiter Abschnitt.

A $\quad k \quad u \quad s \quad t \quad i \quad k$.

12. Physikalisehe Akustik.

A. Masson. Abhandlung über die Geschwindigkeit des 'Jons in den festen Körpern flüssigen und elastischen Fluiden und über die Beziehungen der physikalischen Eigenschaften der

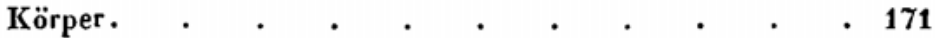

Е. КАнц. Theorie der Luftschwingungen in Röhren . $\quad 174$

Baudrtmon t. Ueber das Ausbleiben der Tonschwingungen in heterogenen Flüssigkeiten. . . . . . . . . 174

H. W. Dove. Eine akustische Interferenz . . . 175

F. G. Schaffвoтsch. Eine akustische Beobachtung . . 176

Trndale. Ueber die chemische Harmonika . . . . 176

O. Schulze. Akustischer Wellenapparat . . . . . $\quad 179$

F. Schafrgorsch. Ueher eine akustische Beobachtung bei der chemischen Harmonika. $. \quad . \quad . \quad . \quad . \quad . \quad 180$

Schrötrвn. Ueber die Ursache des Tones der chemischen Harmonika . . . . . . . . . . 180

G. Stockzs. Wirkung des Windes auf die Intensität des Tones 181

Schaffgotscy. Ueber akustische Versuche . . . . 183

J. J. Opprs. Beobachtungen über eine zweite Gattung von Re-

flexionstönen nebst Andeutungen über die Theorie derselben 186

E. Sane. Theorie der Linearvibration . . . . . 191

Mrister. Akustisches Phänomen . . . . . . 191

F. G. Schafrgotsch. Der Tonflammenapparat . . . 191

Zantemaschr. Ueber die Erzeugung eines dritten Tones durch zwei andere; die Analogie dieser Beobachtung mit den Erscheinungen im Sonnenspectrum . . . . . . 192

Fortschr. d. Phys. XIII. 
Zaxtroeschi. Ueber die Bezielıungen, die zwei zugleich tö-

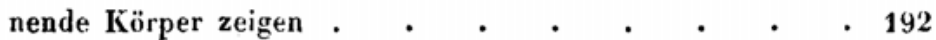

- Ueber die Maafseinheit der musikalischen 'Töne; über die Grenzen der letzteren; über die Dauer der Vibrationen bein akustischen Nerv des Menschen und über die Erhöhung des Grundtons der Stimmgabel, hervorgebracht durch die Molecularveränderung des Metalls . . . . . . 192

Grailich. Ueher singende Flammen . . . . . 193

x. Die amerikanische Dampforgel . . . . 193

13. Physiologische Aknstik. Litératur.

Dritter Abschnitt.

O p t i k.

14. Theoretische 0 ptik.

J.Stran. Allgemeine Gleichungen der oscillatorischen Bewegungen . . . . . . . . . . . . . 197

P. Zвсн. Die Krümmungslinien der Wellenfläche zweiaxiger Krystalle . . . . . . . . . . . . 202

J. E. Prescotr. Ueber die Wellenoberfläche . . . . 206

Sxrder. Ueber die Theorie der kaustischen Flächen, welche in Folge der Spiegelung oder Brechung von Strahlenbüscheln an den Flächen eines optischen Apparats erzeugt werden . 212

J. Perzana. Bericht über optische Untersuchungen. . . 214

G. G. Stock zs. Polarisation des geheugten Lichtes . • . 216

J. Zвсн. Ringsysteme zweiaxiger Krystalle . . . 217

* J. Calandreri. Ueber die Solarrefraction . . . . 217

* O. F. Mossotri. Neue Theorie der optischen Instrumente . 217

* Porno. Vervollkomınung der photographischen Objective;

Discussion des einfachen Objectivs für homogenes Licht . 218

* - Ueber die Theorie der Objective . . . . 218

15. Lichtentwickelung und Phosphorescenz.

E. Becquerel. Untersuchungen verschiedener Lichtphänomene, die aus der Wirkung des Lichtes auf die Körper entspringen 218

D. Vaveran. Ueber das Leuchten der Sonne, der Meteore und der Sternschnuppen . $\quad . \quad$. $\quad . \quad$. $\quad . \quad 220$

Körliker. Ueber die Leuchtorgane der Leuchtkäfer . . 220 
Herapatr. Phosphorescenz der Insekten. . . . 221

A. Vogrz jun. Eine Lichterscheinung durch Reibung 221

16. Spiegelung und Brechng des Lichtes.

W. Hankez. Ueber farbige Reflexion des Lichtes von matt geschliffenen Flächen bei und nach dem Eintritt einer spie-

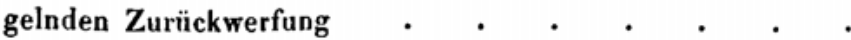

J. Grailich und A. Hande. Note über den Zusammenhang zwischen der Aenderung der Dichten und der Brechungsexponenten in Gemengen von Flüssigkeiten

J. Jamis. Ueber die Messung von Brechungsexponenten der Gase . . . . . . . . . . . . 223

-- Untersuchungen über die Brechungsexponenten . . 227

A. Bren und P. Kremers. Ueber die Brechungsindices einiger

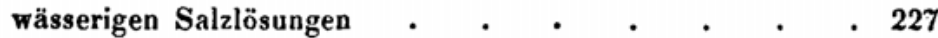

P. Kramers. Brechungsvermögen einiger Salzlösungen . . 228

17. Interferenz des Lichtes.

V. van der Wrlcigen. Constitution der Seifenblasen • . 229

18. Spectrum. Absorption des Lichtes. Objective Farben.

J. H. Gradsrone. Ueber den Gebrauch des Prismas bei der

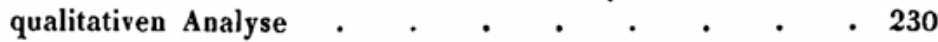

- Das optische Merkmal des Didymium . . . . 230

- Ueber die Farbe der Salzlösungen, bei denen jeder Bestandtheil gefürbt ist . $\quad . \quad$. $\quad$. $\quad . \quad$. 230

- Ueber die Wirkung der Wärme auf farbige Salzlösungen 230

- Ueber die chemische Wirkung des Wassers in Beziehung auf lösliche Salze. . . . . . . . . . . . 230

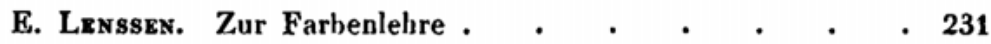

Dovg. Ueber eine Methode, Interferenz und Absorptionsfarben in beliebigem Verhältnifs zu mischen . . . . . 231

J. C. Maxwerc. Experimente über die Farbenwahrnehmung . 232

M. Farndax. Beziehung des Goldes und der andern Metalle zum Licht . $. \quad . \quad . \quad . \quad . \quad . \quad . \quad . \quad$.

J. W. Draper. Ueber das Beugungsspectrum. Bemerkungen zu Eisenlohr's neuen Experimenten • • • • • . 234

W. B. Herapatr. Ueber die optischen Charaktere gewisser Alkaloidverbindungen des Chinins und der Sulfate ihrer Jod-

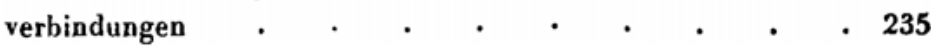

J. W. Malzet. Note über einen Fall der Fluorescenz . $\quad 235$ 
Seite

Govi. Wirkung der Fluorescenzstrahlen auf den Diamanten • 235

J. Grailich. Ueber Fluorescenz . . . . . . 235

Fïrst Salm-Honsтmar. Ueber eine krystallinische Substanz aus der Rinde ron Fraxinus excelsior, welche eine blaue

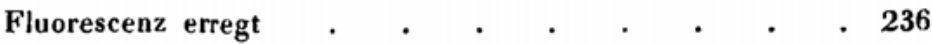

C. M. Guillemin. Ueber die Erscheinung der Fluorescenz . 237

Dove. Ueber das elektrische Licht . . . . . . 237

*A. Fortr. Werthe der Brechungsexponenten einerbeliebigen Substanz in Beziehung zur Wellenlänge des durchgehenden Lichtes

*F. C. Calfert. Ueber Chevreul's Farbenregel . . . 239

* F. Zantedeschi. Ueber die Veränderungen im festen Son-

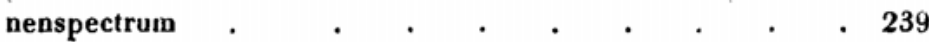

19. Gesehwindigkeit des Lichtes.

20. Photometrie.

F. Zörtnra. Photoinetrische Untersuchungen . . . . 239

B. Srrmiman und C. H. Portra. Note über ein Plotometer und einige Experimente über die relativen Intensitäten mehrerer künstlichen Beleuchtungsmittel . • • • . . 244

Jamin. Die Optik und die Malerei . . . . . . . 245

*T. G. M. Cavallrai. Photometrische Bestimmung der Intensitäten verschiedener Lichtquellen $\quad$. $\quad . \quad$. 245

21. Polarisation. Optische Eigenschaften von Krystallen.

L. Foucault. Neuer Polarisator von Kalkspath • • .

de Senarmont. Ueber die Construction eines doppeltbrechen-

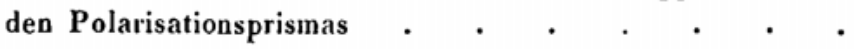

Potrer. Ueber das Princip des Nicol'schen Prismas und über einige neue Formen von Prismen zur Erhaltung linear pola-

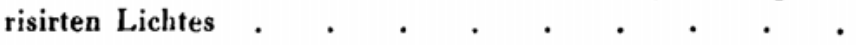

W. Haidingrr. Bemerkungen ïber die krystallographisch-optischen Verhältnisse des Phenakits . . . . . 247

J. J. Ронг. Ueber ein neues Sonnenocular . . . . 248

W. B. Herapath. Untersuchungen üher die Chinin-Alkaloide. 248

Drschorzaux. Ueber die Anwendung der optischen Eigenschaften doppeltbrechender Krystalle auf die Unterscbeidung und Classification krystallinischer Mineralien . . $\quad 249$

G. Søcкоw. Zur Optik der Mineralien ..$\quad$. $\quad . \quad 250$

22. Cireularpolarisation.

Drsclorzadux. Entdeckung der Circularpolarisation in Zinno- 
her, so wie über die gemeinsame Drehung der Polarisations-

Seite ebene durch die Krystalle und die Auflösung des schwefel-

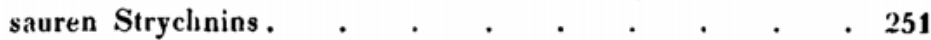

Mirscherlich. Ueber die Mykose, den Zucker des Mutterkorns 253

A. Wurtz. Ueber die Capronsäure. . . . . . 253

\section{Physiologiscbe optik.}

Dove. Ueber Binocularsehen durch rerschieden gefärbte Gläser

- Ueber die Unterschiede monocularer und binocularer Psẹudoskopie

-.. - Darstellung von Körpern durch Betrachtung einer Projection derselben vermittelst eines Prismastereoskops . • 254

A. Cima. Ueber ein neues stereoskopisches Phänomen . 255

J. G. Hazskz. Stereoskop mit beweglichen Bildern . . 255

J. Elz1or. Das 'eleskop-Stereoskop . . . . . . 255

- - Ueber zwei neue Formen des Stereoskops . . . 255

Н. Нвцмногтz. Das Telestereoskop . . . . . . . 256

J. Duв oscQ. Bemerkung ïber ein neues Prismenstereoskop, mit veränderlichem Winkel und beweglichen Linsen . . . 257 van Der Wretrgen. Eine Lichterscheinung in Auge . . 257

Sroutz. Künstliche und mechanische Accommodation des Au-

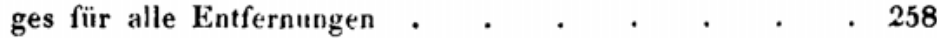

- Zweite Note üher die Accommodation des Auges. . 258

Messens. Untersuchung über die Dauer des Lichteindrucks auf der Retina . . . . . . . . . . . . 258

PaAzzow. Ueber suljective Farben und die Entstehung des

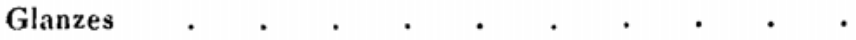

Graud-'Trulon. Bemerkung über den Mechanismus der Hervorbringung cines Reliefs beim Binocularsehen . . .

-C. Bergmanx. Anatomisches und Physilologisches äber die Netzhaut des Auges . $. \quad . \quad . \quad . \quad . \quad . \quad . \quad$.

W. D. Cooner, A. Chauder, D. Brengter, G. H. Lewes. Das Geheimnifs der Umkehr der Bilder im Auge . . . 259

*W. Crookes. Theorie stereoskopischer Bilder . • . 259

*D. Brewster. Das Stereoskop, seine Geschichte, seine Theorie, seine Construction und seine Anwendungen auf Kunst,

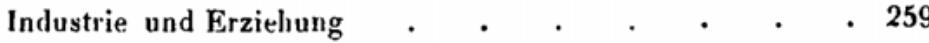

•J. Jago. Ocularspectrum, Einrichtung und Verrichtung . . . 259

* G. Waterston. Ueber ein drittes Instrument derselben Art

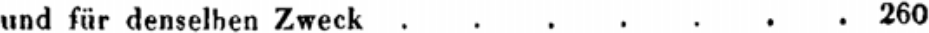


*J. Porno. Ueber Anwendung der pan-fokalen Linse als Oph- Seite thalmoskop . . . .

24. Chemische Wirkngen des Lichtes.

R. Bunsen und H. E. Roscox. Photochemische Untersuchungen 260

J. W. Draper. Einflufs des Lichtes auf Chlor . • . 266

H. E Roscoe. Dasselbe . . . . . . . . . 267

J. W. Draper. Messung der chemischen Action des Lichtes . 267

F. Guтнhг. Wirkung des Lichtes auf Chlorsilber . . . 267

J. J. Watenston. Experimente über die chemische Wirkung

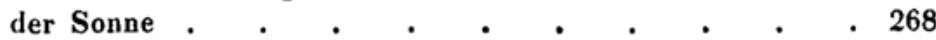

Zantedeschi und Borminetro. Wirkung des Lichtes auf Jod-

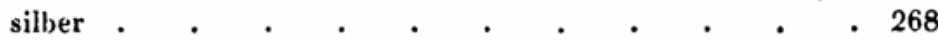

Nièpce de Sarnt-Victor. Ueber eine neue Wirkung des

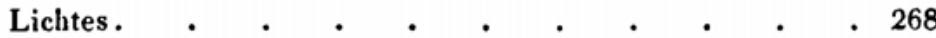

Bertsch. Photographische Bilder mikroskopischer Objective . 270

C. M. Goincemin. Entwickelung des grünen Farbstoffs der Pflanzen und Bengung der Zweige unter dem Einflufs aller

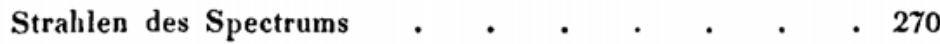

Cherreur. Erklärung des Braun auf den Blättern des Geranium . $. \quad . \quad . \quad . \quad . \quad . \quad . \quad . \quad . \quad .271$

Wissenschaftliche Anwendungen der Photographie. Literatur . . . . . . . . . . 271

25. Optische Apparate.

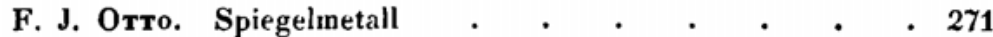

L. Foucaurt. Teleskop mit Silherspiegel • • . $\quad 272$

Sternheil. Ueber die Silherspiegel des Teleskops . . 273

T. Grubs. Verbesserungen an Spiegelteleskopen und Aequatorialinstrumenten . . . . . . . . . . 273

*H. L. Smira. Verbesserung in der Construction des achro-

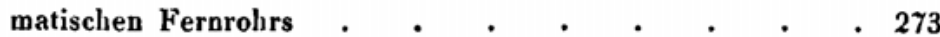

*R. Grezne. Model für eine Maschine zum Polieren der Spiegel von Spiegelteleskopen und der Linsen. . • .

Sole IL fils. Note über die numerische Anordnung der Brillengläser . . . . . . . . . . . . . 273

Porro. Helioskop . . . . . . . . . 274

G. Santrsi. Bemerkung über die Mikrometer, die im dunkeln Felde eines Fernrohrs gebildet sind mit klaren Linsen und

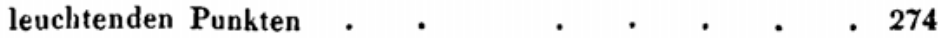

Dкснвr. Beiträge zur elementaren Optik . . . . 274 
P. Casamajor. Methode, Krystallwinkel ohne jedes Gonioneter zu messen

Donovan. Ueher eine bewegliche Sonnenuhr, mit der man Bruchtheile einer Minute bestimınen kanı . . . . 275

D. Brewster. Ueber das Centriren der Linsen von Mikroskop-

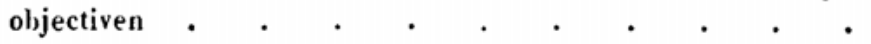
275

'r. W. Wввв. Einfache Methode, die Focallänge kleiner Convexlinsen zu finden . . . . . . . . . . 276

Vierter Abschnitt.

W är m e l e h r e.

\section{Theorie der Wärme.}

*W. Thomson. Ueber die mechanische Energie des Sonnensystems

A. Fuchs. Ueher das Wesen der Wärme und ihre Beziehung zur bewegenden Kraft . $. \quad . \quad . \quad . \quad . \quad . \quad .279$

R. de Napoli. Ueber die Wechselwirkung der Naturkräfte . 279

Séguin. Antwort an Herrn Napoli . . . . . . 279

R. Horpe. Bemerkung zu den Aufsätzen des Hrn. v. Seyolitz und Erwiederung auf die Notiz des Hrn. Crausius betreffend die Wärmetheorie . . . . . . . . 280

F. Mans. Kleine Beiträge zur Undulationstheorie der Wärme 281

R. Clausius. Ueber die Art der Bewegung, die wir Wärme nennen. . . . . . . . . .

J. P. Jodre. Kinige Bemerkungen übser die Wärme und die Constitution elastischer Flïssigkeiten . . . . . .

J. J. Waterston. Ueber die Abweichung von den Grundgesetzen der elastischen Flüssigkeiten, welche sich aus den Versuchen von Regnault und von Thomson und Joule ergeben

W. 'Thomson und J. P. Joun. Ueber die Wärmewirkungen bewegter Flüssigkeiten. 'Temperatur eines Körpers, der sich langsam durch Luft bewegt. . . . . • .

W. Thomson. Ueber die Temperaturrerinderungen, die mit Aenderungen des Druckes in Flüssigkeiten verbunden sind.

J. P. Joule. Ueber die Thermoelektricitait der Eisensorten und über die Wärmewirkung der Ausdelınung fester Körper . 290 
LERovx. 'Temperatur des ausgedelinten Kautschuks

J. P. Jodre. Ueber die Wärmewirkungen der longitudinalen Zusammendrückung fester Körper . . . . . . $\quad$. 291

- Ueber die Ausdelınung des Holzes durch die Wärme . 291

G. Rennie. Ueber die Wärmemenge, die in Wasser durch heftige Bewegung erzeugt wird . . . . . . 293

- - Bericht über die Wärmeerzeugung in bewegtem Wasser 293

J. Trndall. Bemerkungen über Schaum und Hagel . . 294

H. M. Wrtr. Uelıer die Temperatur des Schaumes . . 294

J. Tromson. Ueber die Plasticität des Eises, wie sie sich in den Gletschern zeigt . . . . . . . . . . 295

Hennessx. Ueber das Festwerden der Flüssigkeiten durch Druck . . . . . . . . . . . 295

Calorische Maschinen. Literatur.

27. Wärmeerscheinungen bei chemischen Processen.

Bozrex. Die Heizkraft des Holzgases verglichen mit der des Weingeistes, für die Arbeiten in Laboratorien . . . 297

v. Babo. Argand'sche Gaslampe . . . . . . . 298

28. Physiologische Wärmeerscheinnngen. Literatur. 29. Wärmeleitung.

*Erman. Ueber Boden- und Quellentemperatur und über die Folgerungen, zu denen Beobachtungen derselhen berechtigen

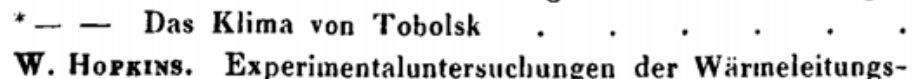
fähigkeit verschiedener Substanzen, mit Anwendung der Resultate auf die Erdtemperatur

30. Specifische und gebundene Wärme.

Grabger. Specifische Wärme roher und plastischer Thonwaaren.

Harrison. Künstliche Fabrikation des Eises . . . . 303

31. Strahlende Wärme.

Zantedeschi. Untersuchung über strahlende Wärme . . 304

R. Franz. Untersuchungen über die Diathermansie einiger gefärbten Flüssigkeiten . . . . . . . . 304

Н. Кловцаисн. Ueber den Einflufs, welchen Metalle auf die strahlende Wärme ausüben . . . . . . . . 307

* A. Sксcнr. Ueber die Flecken und die Temperatur der Sonne. . . . . . . . . . . 312 
*J. W. Enmerins. Ueber die Identität von Licht und strahlen-

Fünfter Abschnitt.

Elektricitätslehre.

\section{Allgemeine Theorie der Elektricităt.}

33. Reibungselektricität.

E. Loomis. Ueber einige elektrische Erscheinungen in den Ver-

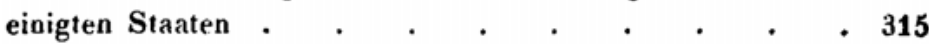

J. Scherder. Dasselbe . . . . . . . . . 315

Eцsнa Foote. Neue Quelle der Elektricität . . . . 316

W. Sirmens. Ueber die elektrostatische Induction und die Verzögerung des Stroms in Flaschendrähten . . . . 316

P. Volpicelli. Elektrostatische Induction . . . . 324

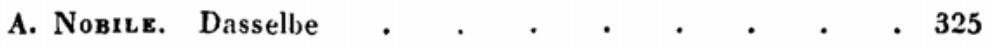

G. Berl. Dasselbe . . . . . . . . . 327

D. R. Fавнт. Kurze Notiz über die Experimente gegen die neue Theorie der elektrostatischen Induction o . 329

'T. Анмеццin. Ein neues Experiment über die elektrostatische Polarität . . . . . . . . . . 330

W. S. HARris. Untersuchungen über statische Elektricität . 330

J. M. Sḱgurs. Fortsetzung einer ersten Reihe von Beobachtungen über die Wirkungen der elektrischen Influenz, in Beziehung mit denen der Induction. . . . . . 330

Rress. Ueber die elektrische Funkenentladung . . . 331

B. W. Frodersan. Beitrïge zur Kenntnifs des elektrischen Funkens . . . . . . . . . . . 333

P. Rızss. Die elektrische Wärmeforınel betreffend . . . 335

W. S. Harkrs. Einige besondere Gesetze der elektrischen Kraft . . . . . . . . . . . . 335

K. W. Knochenhauer. Ueber die Theilung des elektrischen

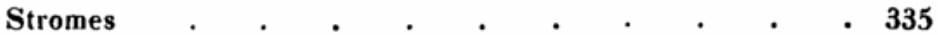

- Ueber den Stroın der Nebenbatterie . . . . . 336

- Beobachtungen über zwei sich gleichzeitig entladende Batterien . . . . . . . . . . 336

P. Voupicelur. Ueber elektrographische Bilder, hervorgebracht mit Hülfe der elektrostatischen Induction . . . . 336 
Morken. Ueber die elektrischen Augenblicksbilder.

Kону. Ueber die Zündung von Minen mittelst des elektrischen

Entladungsfunkens und durch Voxrs'sche Ströme . . 337

C. Bergeal. Notizen über einige elektrische Apparate . . 337 Pyroelektricität.

W. G. Hankel. Thermoelektrische Eigenschaften des Bo-

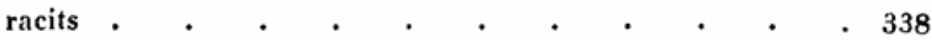

J. M. Gaugain. Elektricität der Turmaline . . . . 339

\section{Thermoelektricität.}

R. Adre. Thermoelektricität des Wismuth und Antimon . . 340

Marbach. Neue Beziehungen zwischen Krystallform und Thermoelektricität

35. Galvanismus.

A. Theorie.

H. Burf. Ueber das elektrische Verhalten des Aluminiums

F. Peтruschersky. Untersuchungen über die Eigenschaften des galvanischen Elementes

Schlagdenhauffen und Frerss. Versuche über die Säule

G. Gone. Erregung der dynamischen Elektricität beim Eintauchen ungleich erwärmter Metalle in Flüssigkeiten

A. Palagi. Ströme, die beim Eintauclien von Kohlen- und Zinkstücken in Wasser entstanden . . . . . 348

Н. JАсовг. Ueber die elektrischen Einheiten . . . . . 349

Lotrner. Zweckmäfsigste Combination einer gegebenen An-

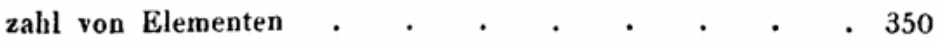

J. Bolzani. Verbreitung des elektrischen Stromes in Körpern 351

J. P. Joule. Schnelzen der Krystalle durch die VourA'sche Batterie . . . . . . . . . .

G. BeцLI. Einzige Möglichkeit gleichzeitiger entgegengerichteter Ströme in einem Leiter

*Jürgensen. Bemerkungen in Beziehung auf die Bewegungen des elektrischen Stromes

B. Galvanische Leitung.

BEñDikr. Abhängigkeit des elektrischen Leitungswiderstandes von der Gröfse und Dauer des Stromes . . . .

W. 'Гномson. Elektrische Leitungsfähigkeit verschiedener käuflicher Kupfersorten . . . . . . . . . .

W. Thоmson. Einflufs der Magnetisirung auf die Leitungsfähigkeit von Nickel und Eisen . . . . . . 
C. Galvanische Ladung und Passivität.

Wrod. Neumann'sche Methode zur Bestimmung der Polarisation und des Leitungswiderstandes . . . . . 357

D. Galvanisches Licht.

Grove. Einige neue Methoden, elektrische Figuren hervorzurufen und zu fixiren . . . . . . . . . . . 360

*'Thury. *E. Wartmans. *E. Becquerel. Elektrische Erleuchtung . . . . .

*Lacassagere und Thirrs. Elektrische Lampe . . . 361

*x. Elektrisches constantes Licht . . . . . . . . 361

*A. Great Gun. Flektrisches Licht . . . . . 361

E. Elektrochemie.

Bertin. Bildung von Wasser durch Platinelektroden . . 362

T. Woods. Ueber die zur Zersetzung chemischer Verbindungen nöthige Zeit . . . . . . . . . . . 363

Osans. Einige zur Elektrolyse gehörige Thatsachen . . 364

H. Burr. Das Verhalten der Chromsäure unter Einwirkung des elektrischen Stromes .

Becquerer. Ueher die langsamen Wirkungen der Wärme und des Druckes . . . . . . . . . . . . 367

L. Cailletrat. Ueber den Einflufs des entstehenden Wasserstoffs auf die Amalgamation. . . . . . . . 367

Osann. Ozonwasserstoff . $. \quad . \quad . \quad . \quad . \quad . \quad . \quad 368$

C. Despretz. Hat es einen Nutzen, bei der cliemischen Zersetzung, einen Inductionsapparat in den Leiter einzuschalten? . . . . . . . . . . . . . . 369

A. DE LA RIVE. Bemerkungen hierzu . . . . . 369

V. Duphḱ. Zerlegung der Salze durch den elektrischen Strom 370

C. Despretz. Zerlegung der Salze namentlich der Bleisalze durch den Strom . .

Martens. Bemerkungen hierzu . . . . . . 371

Wöhlek und Burf. Neue Verbindungen des Siliciuns . . 373

G. Gor в. Moleculare Eigenschaften des Antimon . . . 373

จ. KовеLL. Verhalten der mineralischen Metallsulphurate zur Salzsäure unter galvanischem Einflufs . . . . . $3 \pi 4$

Schlagdenhaupfen. Beobachtungen über einige chemische Zersetzungen mittelst des elektrischen Stromes. . . . 374

F. Galvanische Apparate.

Purvermacher. Constante Säule mit einer Flüssigkeit . . 375 
Osann. Ueber eine Daniell'sche Säule, welche zu Spannungswirkungen gebraucht werden kann . . . . .

F. Prace. U,sache des Kupferniederschlages auf die 'Thonzelle der Danielz'schen Kette und über dessen Verhütung • 375

Bounseus. Ueber den Niederschlag auf den Thonzellen . 376

Kuns. Andere Einrichtung der Kupferzinkkette . . 377

Stonex. Verbesserung der Grove'schen Batterie . . . 378

Bzrozat. Bestimmung derliactoren eines galvanischen Stromes und über einen hierzu sehr bequemen Rbeostaten . . 378

Lacassagne und Thiers. Ueber einen elektrischen Regulator und eine photoelektrische Lampe . $\quad . \quad$. $\quad . \quad 379$

36. Elektrophysiologic. Literatur.

\section{Elektrodynamik.}

G. Кıяснноғғ. Bewegung der Elektricität in Drähten . . 381

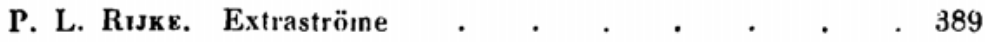

R. Fericr. Versuche äber cinen Fall von Induction, bei welchem die elektrodynamische Wirkung des inducirenden Magnets auf den von einem Strom durcliflossen gedachten Leiter Null wäre . . . . . . . . . .

Bахтвя. Uẹber den Einflufs des Magnetismus auf chemische Wirkung

A. Mecbanische Theorie der galvanischen Kette.

v. Quintus Icruius. Ueber den numerischen Werth der Constanten in der Formel fïr die elektrodynamische Er-

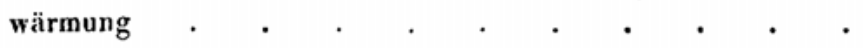

P. A. Favre. Untersuchungen über die durch einen Strom erzeugte Wärme und der chemischen Action des Stromes . 399

J. Bosscra. Ueber die mechanische Theorie der Elektrolyse . 400

L. Soк вт. Ueber die Intensitätsveränderungen, welche der Stroın erleidet, wenn er eine mechanische Arbeit hervorbringt .

- Ueber die Wärmeerzeugung des Stromes in einem Theil der Schliefsung, welcher eine äufsere Arbeit leistet . .

R. Crausius. Bemerkungen über die Beziehung zwischen der chemischen Wirkung, die in einer galvanischen Kette stattfindet und der durch den Strom erzeugten drbeit . . 406

L. Soret. Bemerkungen hierzı . . . . . . 406

F. P. Lenoux. Studien üher elektromagnetische und magnetoelektrische Maschinen . . . . . . . . . 408

R. Chavsius. Elektricitätsleitung in Elektrolyten . . . 409 
38. Galvanisehe Induetion und Magnetoelektricităt.

C. Marteuccs. Ueber ein Phänomen bei einem rotirenden

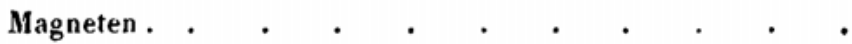

E. S. Riтchre. Abgeänderte Form des Ruhmoorfo'schen In-

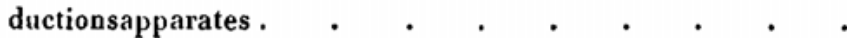

- Ueber den Ruнmкorfa'schen Inductionsapparat . .

L. Fovcaver. Unterbrecher mit doppelter Wirkung für Inductionsapparate . . . . . . . . . . . 414

N. J. Callas. Ueber die elektrodynamische Inductionsmaschine

J. N. Hearder. Ueber ein neues Instrument zur Hervorbringung einer schnellen Aufeinanderfolge von elektrischen Entladungen und äber einen Vergleich der Wirkungen einer Inductionsmaschine mit denen einer Elektrisirmaschine .

Bentlex und Hzarder. Verbesserter Inductionsapparat .

Sinstgden. Ueber die magnetisirende und elektrolytische Wirkung des elektromagnetischen Inductionsstromes . . •

Lenz. Ueber den Einflufs der Geschwindigkeit des Drehens auf den, durch magnetoelektrische Maschinen erzeugten In-

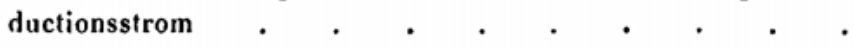

Sizmrss. Uelser eine neue Construction magnetoelektrischer Maschinen . . . . . . . . . . .

Lamx. Anwendung des durch den Erdmagnetismus hervorgebrachten elektrischen Stromes . . . . . .

* A. Noвilz. Ueber ein Fundamentaltheorem der elektrostatischen Induction

39. Elektromagnetismus.

F. P. Le Roux. Ueber den Einflufs der Structur auf die magnetischen Eigenschaften des Eisens. . . . . . . 423

Schefczıк. Abgeänderte Form temporärer Magnete. • . 424

Du Moncer. Versuche über die hinkenden Elektromagnete, die nur mit einer Magnetisirungsspirale versehen sind . . 424

Nıck L̇̀s. Beınerkungen hierzu . . . . . . . 424

DU Moncez. Antwort auf diese Bemerkung . . . . 424

- - Untersuchungen über Elektromagnete . . . . . 426

- Vergleichende Untersuchungen über die Kraft der Elektromagnete, je nach der Stellung ihrer Anker. . . $\quad 426$

- - Ueber die secundären Wirkungen die zwischen den Elektromagneten und ihren Ankern stattfinden . . . 428 
Brgrz. Blektromagnetische Wirkung Voura'scher Ströme ver

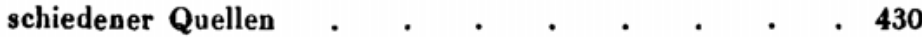

Dus. Ueber die Länge der Elektromagnete . $\quad . \quad$. 431

Mrirzzn. Versuche zur Ausmittelıng des magnetisehen Verhaltens der durch Torsion und Erschütterung veränderten Eisenstangen.

* Romrashauszar. Der verstärkte cylinderförmige Elektromagna 433 Elektromagnetische Maschinen. Literatur.

Wissenschaftliche Anwendungen des Elektromagnetis mus. Literatur.

Fernere Anwendungen des Elektromagnetismus .

40. Eisenmagnetismus.

G. Wrrdrmann. Magnetismus der Stahlstäbe . . . . 436

L. Duroun. Ueber die Abluängigkeit der magnetischen Intensität der Stahlstäbe von ihrer Temperatur . • • .

- Ueber die magnetische Intensität der Elektromagnete, dis über $100^{\circ}$ erwärmt sind . . . . . . . . . 438

J. N. Hrarder. Ueber permanente Gufseisenmagnete . 440

M. Brandikr. Aenderungen des Magnetismus unter dem Einflusse elektrischer Vertheilung . . . . . . 441

H. Kinkzur. Bewegung eines magnetischen Pendels . 442

M. Mzцconi. Magnetische Polarität der Lava . . . 442

- - Ueber die Magnetisirung der Lava in Folge der Wärme und der von der Coercitivkraft einiger magnetischen Gesteine herrührenden Wirkungen . . . . . .

41. Para- und Diamagnetismus.

C. Matrefccr. Experimentaluntersuchungen über den Magnetismus . . . . . . . . . .

Verdet. Ueber die optischen Eigenschaften der magnetischen Körper

$$
\begin{aligned}
& \text { Sechster Abschnitt. } \\
& \text { P h y s i k d e r E r d e. }
\end{aligned}
$$

\section{Meteorologische optik.}

Theoretisches.

M. J. A. Serrer. Ueber eine Stelle der „Mécanique céleste”, betreffend die astronomische Strahlenbrechung 
F. Ralhand. Neue und vollständige Theorie des Regenbogens. . . . . . . . . . . 454

M. F. Sternfunkeln . . . . . . . . $\quad$. 455

*A. Secchr. Sternfunkeln . . . . . . . 455

*E. Iotrneh. Ableitung des Laphace'schen Ausdruckes der atmosphärischen Refraction aus dem Gesetze der Brechung und der Abnahme der Dichtigkeit der Luft mit der Höhe

Beobachtungen zur meteorologischen Optik.
A. Regenbogen, Ringe, Höfe. Literatur.
B. Luftspiegelung. Literatur.
C. Vermischte meteorologisch-optische Beobachtungen. Literatur.
D. Sternschnuppen. Literatur.
E. Feuermeteore. Literatur. Meteorsteine. Literatur.
F. Nordlichter. Literatur. Zodiakallicht.

G. Joses. Beobachıtungen über das Zodiakallicht . . . 459

G. Sonnen- und Mondbeobachtungen. Literatur.

\section{A tmosphărische Elektricităt.}

A. Luftelektricität.

Lamont. Beobaclitung der Luftelektricität zu München . . 461

B. Wolkenelektricität.

a) Ursprung.

A. v. Baumgartark. Ueber Gewitter ïberhaupt, Hagelwetter insbesondere. $. \quad . \quad . \quad . \quad . \quad . \quad . \quad . \quad$. 461

b) Erscheinungen.

M. F. Ueber die dauernde Blitzerscheinung . . . . 4 if2

J. L. Pripson. Ueber einige an der Küste von Westflandern beobachtete meteorologische Erscheinungen. II. Blitze ohne Donner. . . . . . . . . . .

A. Pozx. Bemerkungen über eine Mittheilung des Hrn. PripsoN über die Flächenblitze ohne Donner und die Zickzackblitze mit Donner . . . . . . . . . 463

J. Silbermann. Gewitter vom 18. und 19. Juni 1857 • 463

ЈоваяD. Hypothetische Ansichten über die Ursache des Donners, üher die Wolkenbildung etc. . . . . . 464

*Rümker, E. Bols. Beiträge zur Gewitterkunde . . . 464 
c) Wirkungen.

Guxon. Ueber die am 16. December an Bord der Brigg , la Félicité" durch den Blitz herrorgebrachten Verletzungen .

*W. Prarson. Bericht über ein ungewölınliches Gewitter und über eine zerstörende Localfluth .

A. Porx. Ueber die photographischen Wirkungen des Blitzes

W. Sturgron. Ujeber einige Einzelheiten wibrend eines $\mathbf{G e -}$ wittersturms bei Manchester. . . . . . .

d) Blitzableiter.

* Marchal. Ueber die Apparate, welche in China sich immer auf den Spitzen der Thürme befinden und welche die letzteren nach Art der Blitzableiter vor dem Blitze zu schützen scheinen

H. W. Bruce. Ein vom Blitz getroffenes Schiff . . . 465

W. Sturgen. Ueber Blitz und Blitzableiter . . . . 465

Ozon.

Bérigny. Untersuchungen und praktische Bemerkungen über

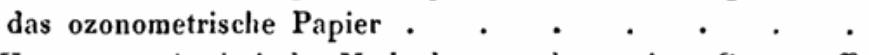

A. Houzenu. Analytische Methode, un den activen Sauerstoff zu erkennen und quantitativ zu bestimmen . . . .

W. Zragr. Ueber eine neue Bestimmungsmethode des Ozonsauerstoffs . . . . . . . . . . . . . 468

*W. B. Rogers. Bemerkungen über's Ozon . . . . 469

*Kornhuber. Ueber das Ozon. . . . . . . . . 469

\section{Erdmagnetismus.}

E. Quetecer. Note über die Bestimmung der magnetischen Declination und Inclination z 1 Brüssel in Jahre 1857.

A. Qurtelex. Note über absolute magnetische Messungen . 469

Sabrna. Ueber die 10jährige Periode in der magnetischen De-

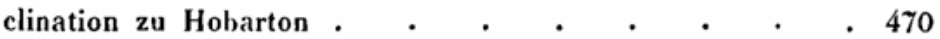

C. А. Sснотт. Ueber die Secularveränderungen der magnetischen Declination an der Küste des atlantischen-Oceans und des Golfes in den vereinigten Staaten aus Beobachtungen

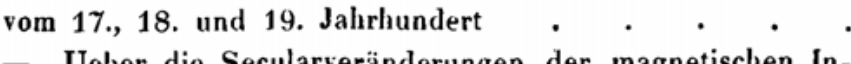

- Ueber die Secularveränderungen der magnetischen Inclination in den nordöstlichen Staaten. . . . . . 471

A. D. Bache und Hirgard. Ueber die Elemente des Erdmagnetismus in den vereinigten Staaten . . . . . 
Scнотт. Magnetische Beobachtungen zu Delaware, Maryland und Virginia . . . . . . . . .

- Versuch, die Secularveränderungen der magnetischen Declination auf der Westküste der vereinigten Staaten zu bestimmen . . . . . . . . . .

- - Versuch, die Secularveränderung der magnetischen Inclination auf der Westküste der vereinigten Staaten zu bestimmen . . . . . . . . . . .

- - Ueber die Secularveränderung der magnetischen Declination an der Küste des atlantischen Oceans und des Golfes in den vereinigten Staaten $\quad . \quad$. . . . . 471

Szcchr. Aufserordentliche Schwankungen der Magnetnadel . 473

Sabznz. Ueber die Leistungen der magnetischen Colonialobservatorien . . . . . . . . . .

Hansteres. Periodische Veränderungen in der magnetischen Inclination in Christiania . . . . . . .

J. F. Encke. Beobachtungen der Declination der Magnetnadel in den Jahren 1847 bis Ende 1854 . . • • • . 476

Sabine. Gröfse und Häufigkeit der magnetischen Störungen und der Nordlichter zu Point Barrow, an den Ufern des

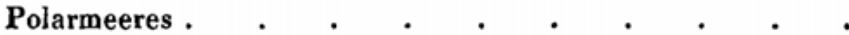

Maguire. Ausbleiben der Störungen der Magnetnadel während der Ausbreitung des Nordlichts . . . . . .

Sabine. Stündliche Beobachtungen der magnetischen Declination durch R. Maguine und die Officiere des Schiffes „Plover" in den Jahren 1852, 1853 und 1854 zu Point Barrow, an den Küsten des Eismeers $\quad$ • . . . . . . . . 477

Hansters. Ueber den Erdmagnetismus . . . . . . 479

M. Wrisss. Declination der Magnetnadel zu Krakau während 17 Jahren . . . . . . . . . . . . 480

H. Tascur. Magnetismus einfacher Gesteine und Felsarten . 480

Haidinger; Schlerermacher. Serpentin mit magnetischer Polarität

*Encke. Magnetische Declination zu Berlin . . . . 481

*P. A. Breton und P. H. J. Boutroz. Anomale Aenderungen der Nadel, beobachtet am 2. April 1857 in den Umgegenden der Insel Ouessant auf zwei verschiedenen Fahrzeugen

*A. B. Frers. Ueber die Aenderung der Nadel . . 
* Yogrl. Ueber die Aenderung der Nadel . . . . 481

* A. D. Bachr. Ueber die allgemeine Vertheilung des Erdmagnetismus in den vereinigten Staaten . . . .

*J. Drummond. Unrifs von einer Theorie der Structur und der magnetischen Erscheinungen der Erde. . . . 481

*A. Sвсcri. Magnetische Beobachtungen. . . . . 481

* - Periodische Aenderung des Erdmagnetismus . . 481

*J. Lanont. Magnetische Beobacistungen angestellt an der Sternwarte bei München in Jahre 1855 . . . .

*__.- Schwankungen der magnetischen Kraft, dargestellt nach den Beobachtungen der Sternwarte l,ei München während der Jahre 1816 bis 1855 . $\quad . \quad$. $\quad$. . . .

*W. Hexzer. Intensität des Erdmagnetisunus in Halle naclı absolutem Maafse. $. \quad . \quad . \quad . \quad . \quad . \quad . \quad$.

*G. Bercr. Vorschlag, die Schiffschronometer vor jeder Unregelmäfsigkeit, durch den Magnetismus hervorgebracht, zı schützen

\section{Meteorologie.}

A. Temperatur.

Ph. Bonleau de Castelnau. Höchste und niederste Temperaturen zu Nînes während 32 Jahren. . . . .

L. Brodger. Vertheilung der Wärme auf der Oberfläche Nordamerikas . . . . . . . . . . . . 483

J. Graisher. Mittlere 'Temperatur für Greenwich . . . 484

J. Srmpson. Temperaturaufzeichnungen für Point-Barrow ans den Jahren 1852 bis 1854 inclusive . . . . .

Hennessy. Berücksichtigung der Seehöhe bei der Bestimmung der Temperaturvertheilung . . . . . . . . 486

- Bemerkung über den Einflufs des Golfstromes auf das

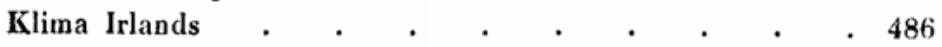

E. Plantamour. Epochen des ersten und letzten Frostes zu Genf . . . . . . . . . . .

H. W. Dove. Ueber die täglichen Aenderungen der Temperatur der Atmosphäre . . . . . . . . .

L. W. Мевсн. Intensität der Erwärmung und der Beleuchtung unter verschiedenen Breiten an der Oberfläche der Erde . 490

W. Lachmann. Die Jahre 1826 und 1846, 1836 und 1856 in ihren meteorologischen Verhältnissen . . . 491

*Y. Kälte zu Dartmouth . . . . • • 494 
*Hennassy. Gleichzeitige Isothermen

*J. P. Harrison. Abhängigkeit der Temperatur vom Mondeinflufs . . . . . . . . . . . 494

${ }^{*}$ E. de WAel. Temperaturbeobachtung . . . . . 494

*Dove. Rückfälle der Kälte im Mai . . . . . 494

*Vessecovskx. Klima von Sitkba . . . . . 494

B. Temperatur und Vegetation.

K. Fritsch. Untersuchungen über das Gesetz des Einflusses der Luftemperatur auf die Zeiten hestimmter Entwickelungsphasen der Pflanzen, mit Berücksichtigung der Insolation

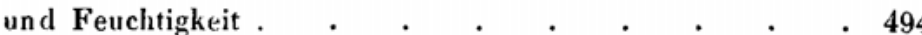

*x. Botanische und zoologische Beobachtungen im Jahr 1855, angestellt zu bestimmten Zeitpunkten . . . . . 497

C. Meteorologisclie Apparate.

Sвсснг. Ueber ein neues Barometer - Luftwage - . 497

- Barometrograph, construirt nach dem Principe der Luftwage . . . . . . . . . . .

Forbes. Geschichtliches über die sogenannte Luftwage, sowie über die Erfindung des Kathetometers. . . . . 499

F. Stach. Wallheim's Thermograph . . . . . 500

Macvicar. Maximum - und Minimumthermometer . . . 500

E. Gand. Einflufs des Erdmagnetismus auf den Index des Maximumthernometers . . . . . . . . . 500

Davour. Neues Barometer . . . . . . . 501

Babret und Davout. Beobachtungen mit demselben . . 501

J. Hentr. Wasserbarometer . . . . . . . 503

S. Stevenson. Selbstregistrirendes Maximum und MinimumHeberbarometer . . . . . . . . . . 503

C. Smallwood. Selbstregistrirender Anemoneter . . . 503

* Viard. Ueber die Reduction auf $0^{n}$ bei barometrischen Höhenmessungen . . . . . . . . . . 503

*A. Quetelet. Instrunente auf dem Brüsseler Observatorium 504

*W. S. Jevons. Ueber Sonnenlanf . . . . 504

*B. Barnabita. Nener meteorologischer Registrator . . 504

*C. Montanx. Ucher die meteorologischen Registririnstrumente und Project zu einer neuen A:t von Instrumenten . 504

*J. Srrbermas. Kautschukballons . . . . . . 504

* Porro. Regenınesser . . . . . . 504

*'Trouessart. Neues Heberbarometer. . . . 504 
*Lapchine. Windrichtuug zu Kharkov, und Beschreibung eines

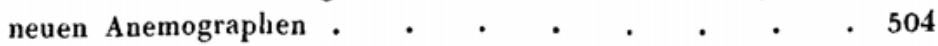

*J. Margurt. Note üher das Bourdon'sche Metallbarometer. 504

D. Allgemeine Beobachtungen.

A. Quetelex. Ueber das Klima Belgiens. 7. Abschnitt . 504

- Meteorologische Beobachtungen aus den Jahren 1853 bis 1855 zu Brüssel . . . . . . . . . . . 504

-.. - Jährliche und stündliche Variationen der meteorologischen Elemente zu Brïssel . . . . . . . . 505

KuprFer. Meteorologische Nachrichten aus Rufsland . . 511

Garle. Die Meteorologie Schlesiens . . . . . 516

J. M. GrLnrs. Resultate der meteorologisch-astronomischen Expedition in die südliche Hemisphäre . . . . . 517

K. Kreil. Meteorologische Untersuchungen für Chartum und Gondokoro . $\quad . \quad$. $\quad . \quad$. $\quad . \quad$. $\quad$. 520

J. Lamont. Meteorologische Untersuchungen für München • 523

Meteorologische Beobachtungen zu Upsala in den Jahren 1855

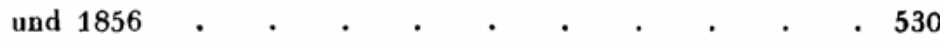

H., A. und R. Schlagintweit. Wissenschaftliche Reisen in Indien und Hochasien . . . . . . . . 530

P. MARÈs. Meteorologische Beobachtungen in der Sahara . 531

Radcliffe. Meteorologisches aus Sinope. . . . . 531

T. S. Parvin. Ueber das Klima von Jowa . . . . 532

E. Vivian. Bemerkungen über das Klima von England. . 534

F. Weber. Meteorologische Resultate für 1856 in Halle. . 534

Ausfecd. Meteorologische Resultate für Schnepfenthal im Jahre

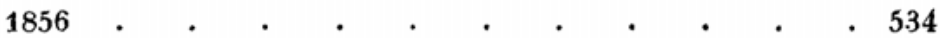

Buxs-Ballot. Ueber die Thätigkeit des niederländischen meteorologischen Instituts. . . . . . . . . 535

Meteorologische Resultate für Lübeck im Jahre 1856 . . 537

*J. F. É́cкr. Meteorologische Beobachtungen von 1847 bis

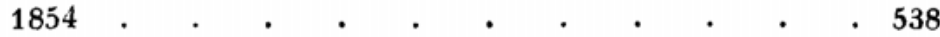

*S. P. HrLderth. Auszug aus einem meteorologischen Journal von Marietta, Olio für 1856. . . . . . . 538

*A. Sесснт. Meteorologische Beobachtungen . . . . 538

*J. Lamont. Meteorologische Beobachtungen zu München für

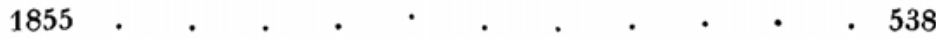

*E. Plantamour. Meteorologisches aus dem Jahre 1856 für Genf und den grofsen St. Bernhard . . . . 538 
*A. Bnown. Auszug aus einem meteorologischen Register für

Seite 1856 zu Arbroath. . . . . . . . . 538

*Listring. Meteorologische Beobachtungen zu Göttingen 1856

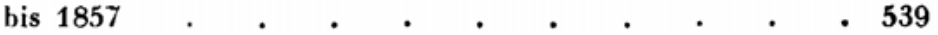

*Prozel Meteorologische Beobachtungen zu Hinrichshagen

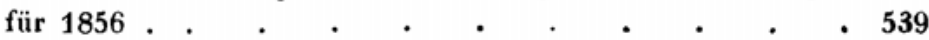

*E. Mrrian. Meteorologische Uebersicht für 1856 . . . 539

*Engemañ und Wiscrzenus. Meteorologische Beobachtungen

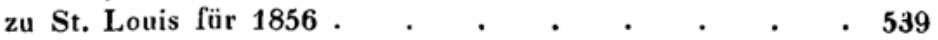

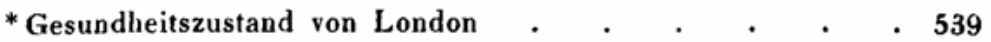

*Dove. Klimatische Verhältnisse des preufsischen Staates . 539

* Meteorologische Beobachtungen in Belgien für 1854 und

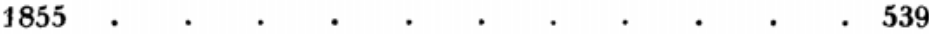

Ausdehnung der meteorologischen Telegraphie . • • $\quad$ • 539

*Babinet. Rückkehr des französischen Klimas auf seinen Nor-

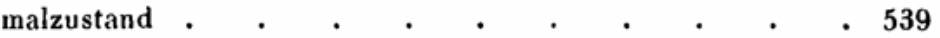

* Kiтtel. Meteorologische Beobachtungen zu Aschaffenburg für

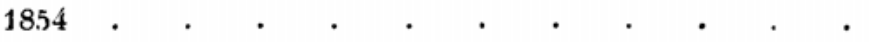

*C. Kunn. Bemerkungen zu den meteorologischen Beobachtungen des Dr. Batr auf seiner Reise im Orient . . 539

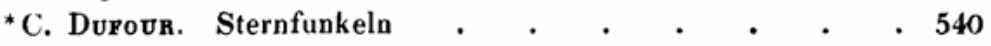

*A. T. Kupfren. Meteorologische und magnetische Beobachtungen in Rufsland für 1855 . $\quad$. $\quad$. $\quad$. 540

- Mittel aus meteorologischen Beobachtungen zu Bogoslovsk, Zlatoouste, Lougan, Novo-Petroosk und St. Petersburg für 1840 bis $1856 \quad$. $\quad . \quad$. $\quad . \quad . \quad . \quad 540$

- Mittel aus meteorologischen Beobachtungen von 15 Jahren zu St. Petersburg . . . . . . . . $\quad$. 540

- Meteorologische Beobachtungen im Staate New-York für

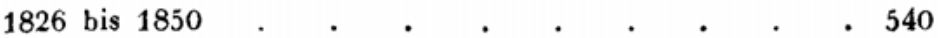

T. LANDson. Meteorologisches Register für 1843 bis 1854 . 541

L. Brodger. Klimatologie der vereinigten Staaten und der gemäfsigten Zone von Nord-Anerika . . . . 541

E. Wind.

Dove. Ueber die allgemeine Theorie des Windes . • . 542

- - Ueber die rom Drelıungsgesetze abhängigen Aenderun-

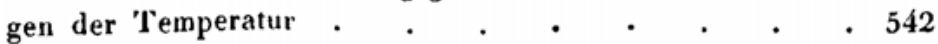

W. C. Redrircd. Theorie der Wirbelwinde und Wirbelstürme 543

-.- Ueber Cyclonenerscheinungen im stillen Ocean . . 544 
F. Vertin. Ueber den aufsteigenden Luftstrom, die Entstehung des Hagels und über Wirbelstïrme . . . . . $\quad 545$

H. W. Dove. Bemerkungen über die meteorologischen Untersuchungen des Hrn. Vetrin . . . . . . 545 Anmerkung des Referenten . . . . . . . 547

*J. Chappelsmith. Verhalten des Barometers bei Orkanen . 548

*Bonnaront. Beobachtungen von Wasserhosen . . . 548

*J. Rodgers und A. Schön bors. Cyclonen auf den Bonininseln 548

* J. Fournet. Winterstïrme in Algerien. . . . . 548

*J. Thomson. Ueber die grofsen Ströme der atmosphärischen

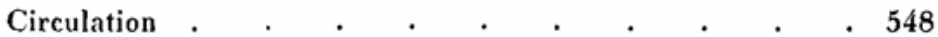

*D. Olmsted. Redrield's Biographie . . . . . 548

* Bache. Ueber die Winde auf der Pacificküste der vereinig-

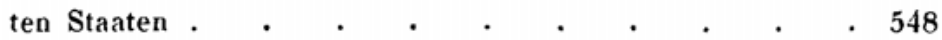

*Henry. Ueber die physikalischen Bedingungen, die das Klima der vereinigten Staaten bestimmen . . . . . 548

${ }^{*}$ G. C. Forshax. Einige Erscheinungen von Texas und Klimatologie . . . . . . . . . . . 548

*Hennessy. Ueber die Verticalströme der Atmosphäre . . 548

${ }^{*}$ W. Tornado in Schuyler . . . . . . . 548

*G. A. Konnhuber. Mittlere Windesrichtung zu Prefshurg . 548

MA URX. Wind - und Stromkarten . . . . . 549

F. Regen, Schnee, Hagel.

H. W. Dove. Vertheilung des Regens auf der Oberfläche der

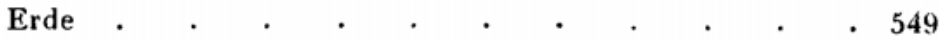

J. Stark. Ueber die Vertheilung des Regens in Schottland . 552

C. Funвооок. Einflufs des Mondes auf die Regenmenge 554

- . Einflufs des Mondes auf die Regenmenge . . . 554

Dalungton. Einflufs des Mondes anf die Regenmenge . . 554

Сн. Martins. Beträchtliche Regenquantität zu Montpellier vom 24. bis 28. September 1857 . $\quad . \quad$. $\quad$ • $\quad$. 554

A. Barthécemx. Pyramidalische Hagelcrystalle mit sechsseitiger ehener Grundfläche . . . . . . . . 555

*T. L. Phipson. Regen oline Wolken zu Paris . . . 555

*Graxsher. Regenfall am 22. October 1857 . . . . 555

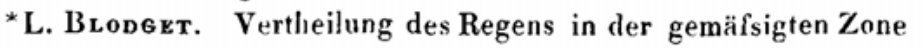

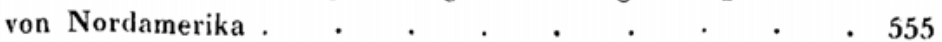

*'T. L. Phipson. Einige meteorologische Phänomene an der Küste des westlichen Flandern . . . . . . . 555 
Seite

*L. Dufour. Regen ohne Wolken . . . . . 555

*Guron. Schlossen von bedeutender Grölse : . . . 555

H. Wolken, Nebel.

*W. S. Jevons. Ueber Federwolken . . . . 555

${ }^{*}$ E. Boll. Rauchende Berge . . . . . . 555

${ }^{*}$ x. Starker Nebel in Paris . . . . . . . 555

${ }^{*}$ Bagot. Nepheleskop . . . . . . . 555

H. Hygrometrie.

J. Luftdruck.

H. W. Dove. Ueher die täglichen Oscillationen des Barometers . . . . . . . . . . . . 556

Grazgrr. Barometerschwankungen und Vegetation . . . 556

J. Lamont. Ueber die Zusammensetzung der Atmosphäre . 556

Burs-Ballot. Beziehung zwischen der Intensität und Richtung des Windes zu den gleichzeitigen Barometerabweichungen. 557

Liagre. Kinflufs des Mondes auf den Barometerstand . 558

F. Vrtrin. Ueber den Barometerstand in verschiedenen Breiten 559

* Boussingault. Beobachtungen üher den Barometerstand im Meeresniveau nahe am Aequator und über die Amplituden der täglichen barometrischen Schwankungen in verschiedenen Höhen in den Cordilleren . . . . . . . . 561

K. B arometrische Höhenmessungen.

J. C. Poggendorfy. Angebliche Ersteigung des Chimborasso . 561

M. C. Dippe. Nichtlogarithmische Tafeln .zur Reduction von Barometerbeobaclitungen auf ein anderes Niveau und zur Bestimmung von Hölenunterschieden aus Barometerbeobachtungen . $\quad . \quad . \quad . \quad . \quad . \quad . \quad . \quad 561$

J. Вӧнм. Ueber die Seehöhe von Prag . . . . . . 561

H. Wolf. Hypsometrische Arbeit vom Juni 1856 bis Mai 1857561

C. Phediger. Beitrag zur hypsometrischen Kenntrif's des Harzes . . , . . . . . . . . . 561

L. Allgemeine 'Theorie.

F. Vetrin. Ueber die Wogen der Luft. . . . . 561

F. Hopkins. Ueber die Wirkung des Wasserdampfes und seine Tendenz, Gleichgewichtsstörungen in der Atmosphäre zu erzeugen .

46. Physikalische Geographie.

A. Allgemeine Beobachtungen.

DaUbRÉE. Untersuchungen über die Streifung der Felsen, Bil- 
dung der Geschiebe, des Sandes und Schlammes und über Seite die durch mechanische Wirkungen hervorgebrachten chemi-

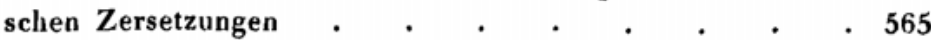

*H. Hopmerster. Chronik der Schweizer Naturerscheinungen 566

B. Meer.

A. Petremann. Der grofse Ocean . . . . . . 566

G. Hagen. Ebbe und Fluth in der Ostsee . . . . 567

*A. Erdmans. Wasserhöhen . . . . . . . 567

C. Irminger. Ueber Ebbe und Fluth im kleinen Belt bei Fri-

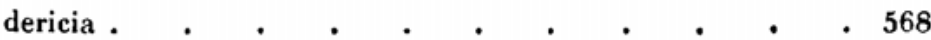

A. D. Bache. Tägliche und halbtägige Fluthen am Golf von Mexiko. . . . . . . . . . . $\quad$. 568

- Fluthöhen an der atlantischen Küste in den vereinigten

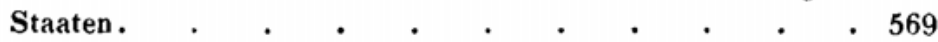

* - Fortschritt in den Fluthtabellen für die vereinigten

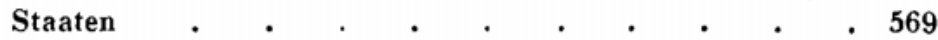

* - Ueber das Anwachsen der Insel Sandy Hook . . 569

Rodgers. Sondirungen im arktischen Ocean . . . . $\quad .569$

T. Spratr. Sondirungen zwischen Malta und dem Archipel . 570

Prince Napolḱon. Meeresströmungen . . . . . . 571

*Wrnne. Ueber den Einflufs des Golfstroms auf das Klima der atlantischen Küste in den vereinigten Staaten $\quad$. 571

J. Le Contr. Thätigkeit des Golfstroms zum Anwachsen von

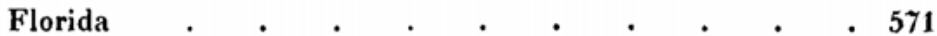

DE Laronce. Versuch, ein allgemeines Gesetz der Strömungen

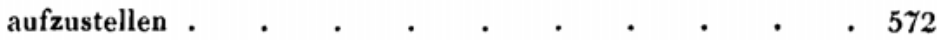

P. P. Krng. Beobachtungen über das specifische Gewicht des Seewassers auf der nördlichen und südlichen Hemisphäre . 572

J. Woucry. Note üher einen durch Eis fortgeführten Block . 573

H. Rıкк. Die physische Beschaffenheit Südgrönlands • . 573

E. K. Kank. Polarforschungen . . . . . $\quad$. 574

C. Seen.

L. L. VALLÉE. Note über den Genfer See . . . . 574

C. Whiтlesex. Ueber die Veränderungen des Niveaus der grofsen nordamerikanischen Seen . . . . . . . 574

Strabriowski. Das Phänomen der Seiches in Onegasee . 575

D. Quellen.

C. Bromers. Das Geysirphänomen imitirt durch einen Apparat nach Bungen's Geysirtheorie . . . . . . $\quad$. $\mathbf{5 7 6}$ 
Seite

Bornemans. Ueber die eruptiven Phänoınene Sardiniens . 576

_ _ Mineralquellen Sardiniens . . . . . . . 577

C. Ladrent. Artesische Brunnen der östlichen Sahara . . 577

*A. B. Nonтнсоте. Die Salzquelle von Cheshire . . . 577

*Ville. Salzquelle, Mineralwasser etc. in Algier . . . 577

*'T. Simmцer. Alkalisches Schwefelwasser in Glarus . . 577

*Landerer. Heilquellen von Kaiapha im Peloponnese . . 577

${ }^{*}$ Guron. Mineralwasser in Tunis . . . . . . 578

*R. Fresenius. Mineralquelle in Weilbach . . . . 578

*E. E. Lang. Das Trentschin-Teplitzerthal und dessen Mine-

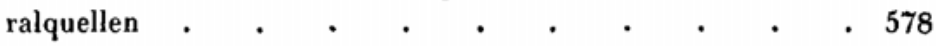

E. Flüsse.

R. Schlagintweit. Erosionsformen der indischen Flüsse . 578

J. Lamont. Temperatur der Isar und der am rechten Isarufer befindlichen Quellen. Beobachtet von 1852 bis 1856 . 578

H. W. Dove. Ueher die Wärme der Flüsse . . . . 578

E. S. SNrzl. Vibrationen des Wassers am Wasserfall bei Halyoke, Massachusetts .

Simonx. Alluvialgebilde des Etschthales . • . . 581

- - Ueberschwemmung des Vintschgau. Sommer 1855 . 581

P. Charx. Beobachtungen über das Arve- und Rhonegebiet . 581

T. Logan. Ueber das Delta des Irrawaddy . • . . 582

*J. Franz. Beobachtungen über den täglichen Wasserstand des Nils vom April bis August 1857. . . . . . 583

F. Gletscher.

J. Trndall und J. H. Huxcex. Structur und Bewegung der Gletscher . . . . . . . . . . . 583

T. H. Huxcex. Structur der Gletscher . . . . . 585

S. BAup. Ueber den Grund des Fortrückens der Gletscher . 587

ZoLLIKofer. Macugnagagletscher . . . . . . $\quad . \quad 587$

K. v. Sonkla . Der Ausbruch des Suldnergletschers in 'Tyrol 587

EцEкт. Gletschersturz bei Randa in Visperthal an 31. Januar 1857 . . . . . . . . . . . . . 588

*J. BALL. Gletscherstructur . . . . . . . . 588

${ }^{*}$ A. Baver. Ueber Gletscher . . . . . . . 588

*E. Colxomb. Ueber Gletscher . . . . . . 588

G. Bodentemperatur.

R. W. Fox. Temperatur einiger Minen in Cornwall $\quad$. 589

A. Litron. Artesischer Brunnen in St. Louis . . . $\quad$. 589 
Schwan. Ueber ein Phänomen der Erdtemperatur . . 589

Walfradis. Neue Untersuchungen über die Erdtemperatur in grofsen Tiefen . $\quad . \quad$. $\quad . \quad$. $\quad . \quad 590$

H. Gasentwicklung.

x. Ausströmung von brennbarem Gas ats der Erde . . 590

J. Senkung des Landes.

G. H. Coок. Senkung des Landes an der Seeküste von NewJersey und Long Island . . . . . • • 591

K. Berge.

WARD. Der Gebel-Nakus oder Glockenberg . . . 591

L. Vulcane und Erdbeben.

Palmieri. Vesuy . . . . . . . . . . 591

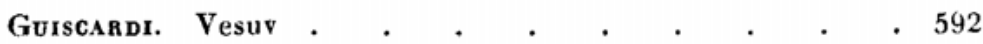

L. Palmieri. Einige Beobachtungen ïber die 'Temperaturen der Fumarolen, die sich auf der Lava des Vesurs bilden . 595

- Meteorologische und physikalische Beobachtungen während des Ausbruchs des Vesuvs im Mai 1855 . $\quad . \quad 596$

Авıсн. Lichterscheinungen auf dem Kraterplateau des Vesurs im Juli 1857 . $\quad . \quad$. $\quad . \quad$. . . . . 597

F. D. Hartrand. Der Vesuv und seine Eruptionen . 597

* J. Rorн. Der Vesuv und die Ungebung von Neapel . . 597

P. Semenow. Ueber vulcanische Erscheinungen in Central-

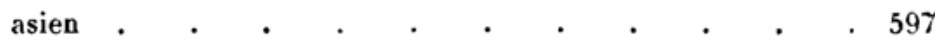

A. Jansen. Ausbruch des Awoe auf der Insel Sangir den 2.

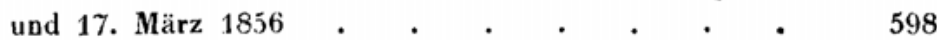

'T. Coan. Vulcaniscbe Thätigkeit in Hawaii . . . . 598

*C. T. Wrsslow. Ueber die vulcanischen Plänomene des Kilauea und Mauno Loa . . . . . . . . 599

H. Karsten. Ueber die Vulcane der Anden . . . . 599

C. $x$. Ein neuer submariner Vulcan . . . . . 599

J. B. Trask. Erdbeben in Californien während-des Jahres 1856 . . . . . . . . . . . 599

A. Cousin und A. H. Matrigu. Unterseeischer Vulcan an dem Aequator, zwischen dem 20. und 22. Grad westlicher Länge 600

G. Jones. Ueber einen Aschenfall in der Ebene von Quito . 600

Bonsemas. Bericht äber eine Reise in Italien . . . 600

Burkarr. Ein neuer Feuerausbruch im Gebirge von Real del monte in Mexico. . . . . . . . . . . 601

* - Ueber die Erscheinungen bei dem Ausbruche des me- 
xikanischen Feuerberges Jorullo im Jahr 1759, nebst Zusatz von A. ₹. Humbold .

C. S. C. Deville. Ueber vulcanische Emanationen . . . 601

C. S. C. Deville und F. Leblanc. Ueber die chemische Zusammensetzung der aus den vulcanischen Gegenden Süditaliens ausströmenden Gase $\quad \cdot \quad \cdot \quad \cdot \quad \cdot \quad \cdot \quad$.

Guiscardr. Note üher die Gasausströmungen der Phlegräischen

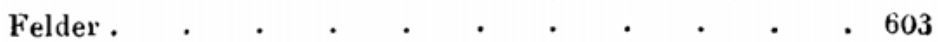

C. S. C. Deville und F. Leblanc. Ueber die Gasausströmung in den toskanischen Borsäurefumarolen . . . . . 604

Palmieri. Bemerkung über einen elektromagnetischen Seismographen . . . . . . . . . . . 604

K. J. Clement. Die ringförmige Bahn der Erdbeben . . 605

E. Kluge. Beleuchtung von Chement's Theorie der Erdbeben 605

*A. Bouk. Parallele der Erdbeben, der Nordlichter und des Erdinagnetismus sammt ihrem Zusammenhang mit der Erdplastik sowohl als mit der Geologie . . . . .

${ }^{*}$ E. Kuvgr. Verzeichnifs der Erdbeben und vulcanischen Eruptionen und der dieselhen begleitenden Erscheinungen in den Jahren 1855 und 1856. . . . . . . .

A. Percx. Note über die in Jahre 1855 bemerkten Erdbeben, mit einem Supplement für die früheren Jahre . • .

Tscheinen. Tagebuch über die Erdbeben des Visperthales in den Jahren 1855 und 1856 . $. \quad . \quad . \quad . \quad . \quad .605$

E. Roвентs. Erdbeben . . . . . . . . 606

Wurzer. Erdbeben in Brussa. . . . . . . 606

G. Dolsfuss. Wirkung des Erdbebens vom 25. Juli 1855 an der Sitterbrücke hei St. Gallen . . . . . . 607

Tschernen. Felssturz bei Grächen . • • • . . 608

Heusser. Analyse des Wassers zweier in Folge des Erdbebens im Visperthal neu entstandenen Quellen . . . 608

DaubréE. Südgrenze des Erdbebens im Elsals am 25. Juli 1855

de Stanarmon. Erdbeben in Algerien vom 22. August bis 15. October 1856.

Гrost. Erdbeben in Nizza. October 1856 bis September

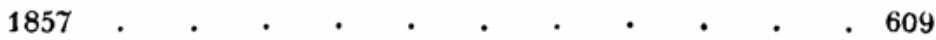

Nöggerath. Erdbeben in Siebengebirge. 6. December 1856610

A. BrRt. Erdstols in Venedig . . . . . $\quad$. 610 
Coxla. Erdbeben in Parma am 31. Januar 1857 . Seite Muston. Erdstofs in Montbéliard . . . . . . . 612

H. Lecoce. Erdbeben in Clermont-Ferrand an 16. Juni 1857612

Greber. Erderschütterung in Sachsen und Thüringen . . 613

*H. Hormerstre. Chronik der in der Schweiz beobachteten Naturerscheinungen . $. \quad \cdot \quad . \quad$. $\quad$. 613

Namen - und Capitelregister . $\quad . \quad$. $\quad . \quad$. $\quad . \quad 6614$

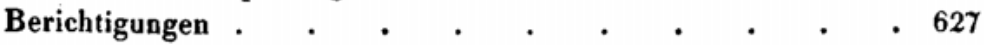

Verzeichnifs der Herren, welche für den vorliegenden Band

Berichte geliefert haben $. \quad . \quad . \quad . \quad . \quad 628$ 